\title{
Unexplained Variation in Benefit of Treatment of Congenital Unilateral Aural Atresia: A Review of the Literature
}

\author{
Katharina Vogt ${ }^{a}$ Jolien Desmet ${ }^{b}$ Arno M. Janssen ${ }^{b}$ Martijn J.H. Agterberg ${ }^{a, b}$ \\ Ad F.M. Snik ${ }^{a}$ \\ ${ }^{a}$ Department of Biophysics, Donders Institute for Brain, Cognition and Behaviour, Radboud University Nijmegen, \\ Nijmegen, The Netherlands; 'bepartment of Otorhinolaryngology, Donders Institute for Brain, Cognition and \\ Behaviour, Radboud University Medical Centre Nijmegen, Nijmegen, The Netherlands
}

\section{Keywords}

Aural atresia - Binaural hearing $\cdot$ Conductive hearing loss · Congenital hearing loss $\cdot$ Treatment

\begin{abstract}
Objective: A review of published data regarding binaural hearing after treatment of congenital unilateral conductive hearing loss (UCHL) due to aural atresia. Treatment options concern atresia surgery (reconstructive surgery), application of a bone conduction device (BCD), or application of a middle ear implant (MEI). Data Sources: Database PubMed was searched for articles published in English and German between January 1, 1994, and January 1, 2019. Study Selection: The initial search identified 52 studies, of which 9 met the inclusion criteria. Data Synthesis: Comparison of studies was based on a structured review. Meta-analysis was not feasible because of the heterogeneity of outcome measures, the limited number of relevant papers (9), and diverse types of treatment (5). Conclusions: Treatment of UCHL results in bilateral hearing instead of binaural hearing. The large intersubject variability in benefit of treatment is unexplained with a clear improvement in the minority of listeners and a limited improvement or binaural interference in most listeners after atresia repair or amplification with a BCD or $\mathrm{MEI}$.
\end{abstract}

(c) 2021 The Author(s).

Published by S. Karger AG, Basel

karger@karger.com www.karger.com/aud

Karger $\stackrel{\text { ' }}{5}$

GOPEN ACCESS
(C) 2021 The Author(s)

Published by S. Karger AG, Basel

This is an Open Access article licensed under the Creative Commons Attribution-NonCommercial-4.0 International License (CC BY-NC) (http://www.karger.com/Services/OpenAccessLicense), applicable to the online version of the article only. Usage and distribution for commercial purposes requires written permission.

\section{Introduction}

Studies have shown that children and adults with unilateral hearing loss might encounter serious problems in daily life, as 2 ears are essential for proper directional hearing and recognition of speech in environments with competing sounds [Bess et al., 1984; Lieu et al., 2012; Lieu, 2013; Sangen et al., 2017]. In addition, children with unilateral hearing loss might be prone to delayed speech development and learning difficulties in school affecting academic achievements [Kesser et al., 2013; Kuppler et al., 2013]. Children with unilateral conductive hearing loss (UCHL) tend to do better overall (school and behavior) than children with unilateral sensorineural hearing loss [Kesser et al., 2013].

One cause of UCHL is congenital aural atresia, which occurs in approximately 1 per 10,000 live births [Alasti and van Camp, 2009]. Congenital aural atresia is unilateral in approximately $70 \%$ of affected children. Nowadays, rehabilitation options for these subjects are either surgical repair or fitting of a hearing device, like a bone conduction device (BCD) or a middle ear implant (MEI). What type of hearing device is used, or whether a device is used at all, depends on multiple factors, for example, preference of the clinic/country and coverage by health insurance but also the age at intervention and anatomy of the atretic middle ear.

Martijn J.H. Agterberg

Donders Institute for Brain Cognition and Behaviour, Biophysics

Heyendaalseweg 135

NL-6500 Nijmegen (The Netherlands)

m.agterberg@donders.ru.nl 
In general, the aim of such treatments is to improve hearing in the operated/treated ear with the ultimate goal to provide the patient access to binaural cues to be able to overcome aforementioned listening limitations. Binaural cues are, for example, essential for accurate horizontal directional hearing, which is based on the detection of interaural difference cues, and crucial for solving the "cocktail party" problem [Blauert, 1997; Bronkhorst, 2000; Carlile et al., 2005]. When 1 ear is occluded, access to interaural differences is distorted, jeopardizing directional hearing in azimuth.

Assessing binaural hearing abilities after treatment is fundamental to evaluate the benefits of hearing rehabilitation in patients with UCHL. Several measures can be used to document binaural advantage, namely directional hearing ability, speech recognition with spatially separated speech and noise sources, and binaural summation [Avan et al., 2015; Snik et al., 2015]. Assessment of horizontal sound localization is appropriate for the evaluation of binaural hearing [Kitterick et al., 2011; Snik et al., 2015]. Speech recognition with spatially separated speech and noise sources is mainly based on the acoustic head shadow, which is the underlying physical concept of interaural level differences (binaural cue). Head shadow refers to the attenuation of sounds by the head and torso and, for broadband sounds like speech, amounts to $4-5 \mathrm{~dB}$ [Kunst et al., 2008; Agterberg et al., 2011]. Head shadow might result in a better speech-to-noise ratio (SNR) for one of the ears depending on the positions of speech and noise sources [Plomp and Mimpen, 1981; Bronkhorst and Plomp, 1988]. Different positions of these sources also lead to interaural phase differences for the noise and speech signals, which might further improve the SNR, referred to as binaural squelch [Levitt and Rabiner, 1967; Avan et al., 2015]. Binaural summation refers to louder perception of sounds or speech when listening with 2 instead of 1 ear. In quiet, binaural summation accounts for 4-6 dB [Dillion, 2012; Avan et al., 2015] while binaural summation-in-noise accounts for $1.5 \mathrm{~dB}$, maximally [Davis et al., 1990].

Application of a percutaneous BCD to enable a bilateral auditory input has shown to improve speech recognition in noise and spatial hearing in subjects with congenital [Kunst et al., 2008] and acquired UCHL [Agterberg et al., 2011; Agterberg et al., 2012]. Similar results were seen with an active transcutaneous BCD [Vyskocil et al., 2017; Magele et al., 2019]. Concerning the MEI, Frenzel et al. [2009, 2015] showed that hearing and speech recognition of the impaired ear of children with unilateral CAA improved significantly; however, binaural hearing was not tested.
However, the main question that has to be answered is, can the auditory information from the treated atretic ear complement the normal-hearing ear, leading to binaural hearing (thus effectively "fusing" the 2 inputs)? This question has not been discussed in detail in previous studies.

Therefore, the aim of this study is to address this question by presenting a systematic review of published data with specific emphasis on binaural hearing outcomes after treatment (either reconstructive surgery or application of a hearing device) of subjects with congenital UCHL owing to aural atresia.

\section{Methods}

\section{Search Strategy}

This review follows the PRISM protocol (Preferred Reporting Items for Systematic reviews) [Moher et al., 2009]. As database, MEDLINE (PubMed) was used. The review specifically addresses binaural hearing outcomes after treatment of congenital UCHL. As search terms, we used "unilateral" and "conductive," "atresia" or "congenital," and "aural" or "ear." These terms were combined with "binaural," "directional," "localization," or "speech." The search syntax is shown below. Only papers written in English or German were included. The search interval covered the last 25 years, from January 1, 1994, to January 1, 2019.

The search string is as follows: $((()(($ unilateral AND conductive AND ((atresia AND (aural OR ear)) OR congenital)) AND (binaural OR directional OR (localization OR localization) OR speech))) AND (English[Language] OR German[Language]))) AND ("1994/01/01"[Date - Publication] : "2019/01/01"[Date - Publication]))).

\section{Study Selection and Methodological Quality}

Studies were selected if they fulfilled the criteria, based on the title and abstract. If appropriate, the full article was retrieved and judged. We screened references of these articles to verify whether or not we missed other relevant papers.

The quality of studies was assessed based on different categories: ethical approval, prospective study, eligibility criteria specified, a power calculation made, appropriate controls and outcome measures used, confounding factors reported and controlled for, appropriate analysis made, and any missing data accounted for.

Extracted data from the included studies comprised number of subjects, time of follow-up, type of pathology, type of treatment, outcomes, and whether reference (normative) data were available. Outcomes comprised posttreatment hearing thresholds, binaural summation scores, speech perception in noise scores with spatially separated speech and noise sources, and directional hearing.

\section{Comparison to Normative Data}

If available, the posttreatment scores were compared to normative (normal-hearing listeners) data. This enabled us to express the binaural scores as Cohen's $d$ effect size, using the standard deviations of the normative data (equation (1)). In equation (1), $\mu_{\text {pat }}$ and $\mu_{\text {norm }}$ represent the populations' mean of the patients and the nor- 
mal-hearing listeners, and $\mathrm{sd}_{\text {pat }}$ and $\mathrm{sd}_{\text {norm }}$ stand for the populations' standard deviation of the patients and normal-hearing listeners. If the effect size $d$ was $\leq 0.2$, then the effect (difference between the patients and normal-hearing listeners) is large; if $d$ was $\geq 0.8$, then the effect is small [Cohen, 1988].

$$
d=\frac{\mu_{\mathrm{pat}}-\mu_{\mathrm{norm}}}{\frac{\sqrt{\left(\mathrm{sd}_{\mathrm{pat}}^{2}\right)+\mathrm{sd}_{\mathrm{norm}}^{2}}}{2}} .
$$

\section{Results}

\section{Study Selection and Characteristics}

The search resulted in 55 studies. Papers that clearly appeared to have no relevance to the research question were excluded, for example, papers addressing bilateral aural atresia $(n=6)$ and other study populations $(n=5)$ like acquired hearing loss. Next, based on the abstracts, papers that were not addressing binaural outcomes or that had fewer than 5 subjects $(n=22)$ were excluded, as well as reviews, guidelines, and "current opinion" papers $(n=13)$. One study presenting animal research was excluded as well (Fig. 1).

After a full text review, 8 studies were found. Screening the references of these studies led to inclusion of 1 more relevant paper [Leinung et al., 2017]. The included 9 studies describe together results in 139 treated subjects with unilateral congenital aural atresia (except 3 of the 19 patients in Wilmington et al. [1994], 2 of 20 in Kunst et al. [2008], and 1 of 12 in Nelissen et al. [2016], who had a congenital middle ear anomaly). All studies comprised prospective, non-randomized intervention studies. Power calculations were not made in any of the papers; appropriate control data or reference values were included or referred to in 5 out of the 9 studies. The outcome measures were well defined in all the studies, and appropriate descriptive statistical analyses were made.

Five papers presented data compiled after atresia repair [Wilmington et al., 1994; Gray et al., 2009; Moon et al., 2014; Byun et al., 2015; Kesser et al., 2016] (altogether 93 subjects) and 4 papers after application of a hearing device, a percutaneous BCD (Baha ${ }^{\circledR}$ Cochlear Bone Anchored Solutions [BAS], Gothenburg, Sweden [Kunst et al., 2008; Nelissen et al., 2016]), an active transcutaneous BCD (Bonebridge ${ }^{\mathrm{TM}}$; MED-EL, Innsbruck, Austria [Vyskocil et al., 2017]), a passive transcutaneous BCD (Sopho$\mathrm{no}^{\mathrm{TM}}$; Medtronic, Jacksonville, USA [Nelissen et al., 2016]), or an MEI (Vibrant Soundbridge ${ }^{\circledR}$ [VSB]; MEDEL, Innsbruck, Austria [Leinung et al., 2017]). In total, 46 subjects were treated with a hearing device; obviously, in all cases, it comprised a semi-implantable hearing device.
Total number of potentially relevant publications using search strategy: 55

(((()((unilateral AND conductive AND ((atresia AND (aural OR ear)) ORcongenital)) AND (binaural OR directional OR (localization OR localization) OR speech))) AND (English[Language] OR German [Language]))) AND ("1994/01/01"[Date - Publication] : "2019/01/01"[Date - Publication])))

Number of publications excluded with reasons:

- Review / Guidelines = 13

- Bilateral aural atresia $=6$

- Different study population = 5

- Required study population but different outcome measures or number of patients $<5=22$

- Animal studies $=1$

Number of publications added with reasons: - Screening of references $=1$

9 publications selected for inclusion in systematic review

Fig. 1. Flowchart of study selection. Search in database MEDLINE (PubMed) resulted in the identification of 55 publications with 1 publication added due to screening of references.

\section{Outcome Measures}

\section{Posttreatment Hearing}

Table 1 gives an overview of the included studies. The degree of asymmetry in hearing after the treatment refers to posttreatment hearing thresholds of the impaired ear minus the air conduction hearing thresholds of the normal-hearing ear. Several studies only presented posttreatment thresholds, while the better ear was stated to have "normal hearing." For these studies, only posttreatment hearing data are presented. The last column of the table indicates whether or not normative data were available.

\section{Directional hearing}

In 6 studies, outcomes of directional hearing experiments were reported. Different experimental setups were used regarding the type of stimulus (broad-band or narrow-band noise), the length of the stimuli (150 ms to $2 \mathrm{~s}$ ), the use of sound level (roving), arrangement of the sound sources (loudspeakers), and the total number of presented sound stimuli. Three different outcome measures have been used, namely percentage of correctly identified sound sources; the mean absolute error (MAE), which is the absolute difference in degrees between the target sound source and the identified sound source; and the root-mean-square value of the localization errors. 
Table 1. Some characteristics of the included studies

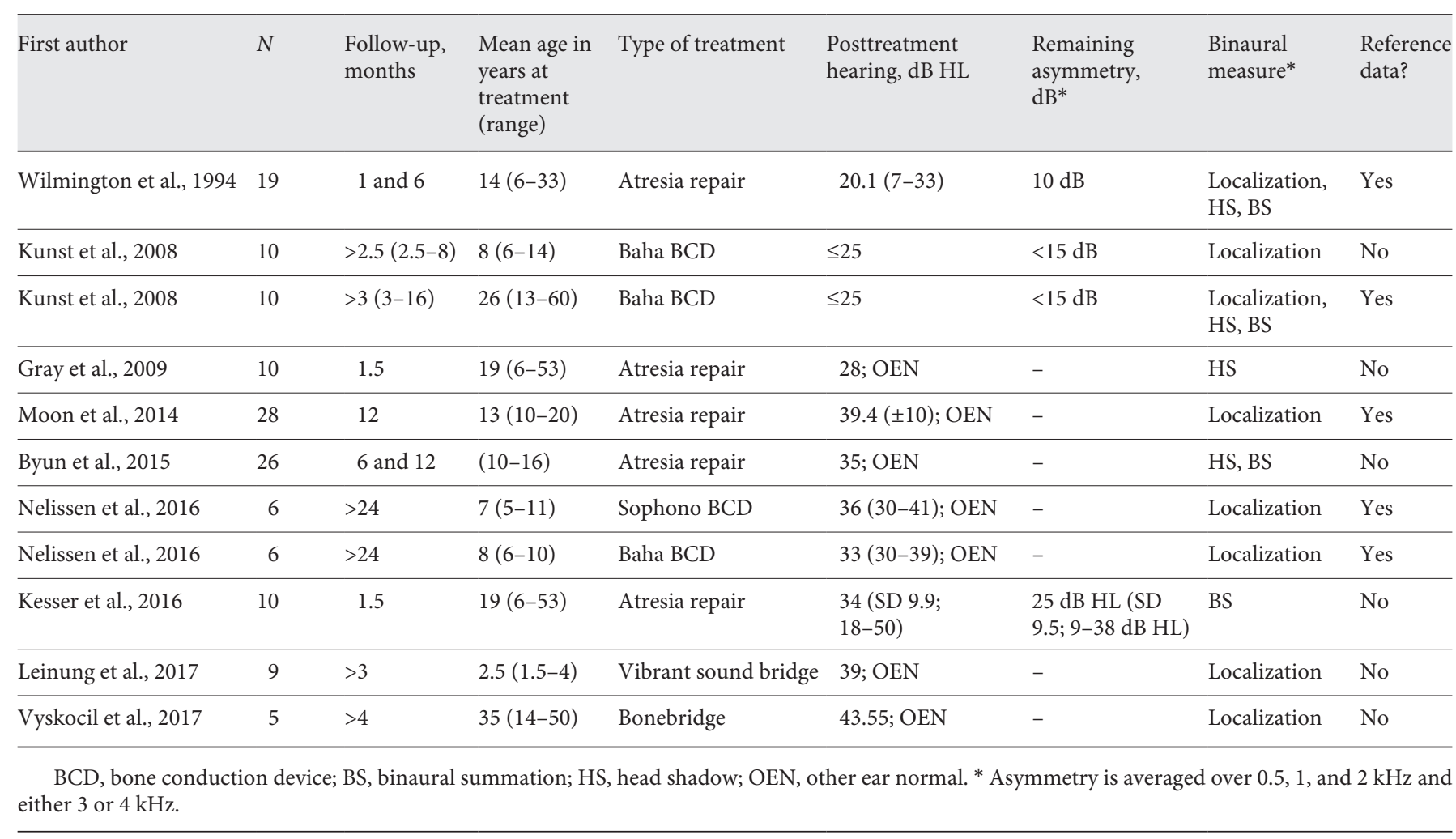

Table 2. Directional hearing data from 6 studies

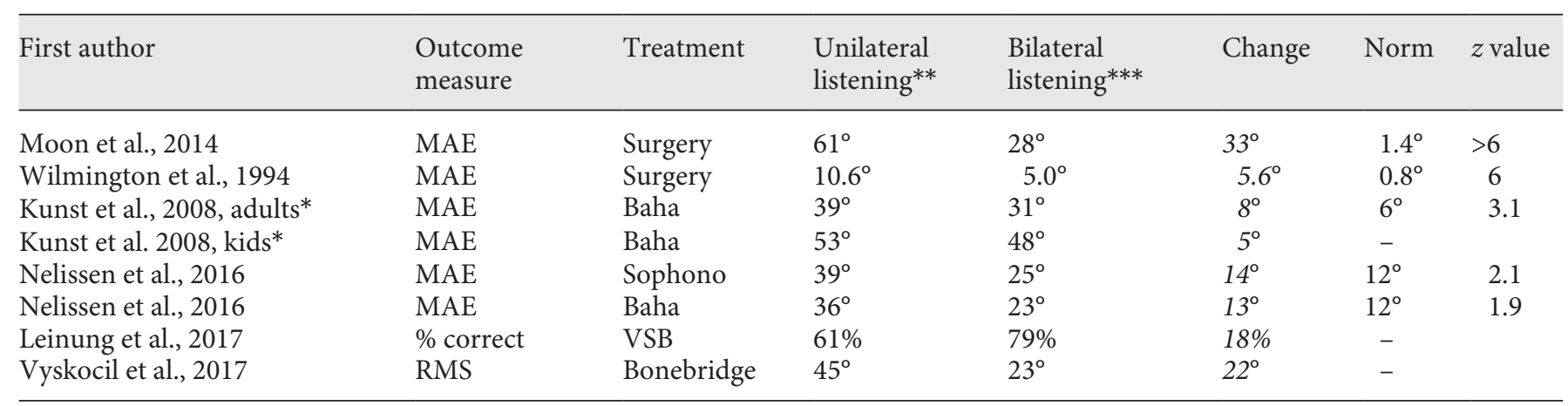

MAE, mean absolute error; RMS, root-mean-square value of the localization errors. * Data from experiments with high-frequency noise bursts. ${ }^{* *}$ Unilateral listening refers to either the situation pre-atresia surgery or the unaided situation. ${ }^{* * *}$ Bilateral listening refers to the posttreatment situation.

Table 2 presents the outcomes of the directional hearing tests. It should be noted that Nelissen et al. [2016] compared 2 groups of subjects, using either a percutaneous BCD (Baha ${ }^{\circledR}$ ) or a passive transcutaneous BCD (Sophono $\left.^{\mathrm{TM}}\right)$. Kunst et al. [2008] presented the results obtained in children and adolescents/adults separately, owing to different measurement protocols.
Variable results in the studies are presented in Table 2, which might at least be partly ascribed to differences in experimental conditions. Therefore, we considered the "change" between the scores (bilateral minus unilateral score), which is less dependent on the experimental setup. In addition, the Cohen's $d$ effect scores are also much more robust against different measurement conditions. 
Table 3. Results of binaural summation tests in 4 studies

\begin{tabular}{llll}
\hline First author & Treatment & BS in quiet & BS in noise \\
\hline Byun et al., 2015 & Atresia repair & & $0.8 \mathrm{~dB}(\mathrm{~ns})$ \\
Kunst et al., 2008, adults & Baha $^{\circledR}$ & 1.3 , range -1.2 to $+5.9 \mathrm{~dB}(\mathrm{~ns})$ & \multirow{2}{*}{$0.85 \pm 2.9 \mathrm{~dB}$ (ns) } \\
Kesser et al., 2016 & Atresia repair & $3.4 \pm 7.6 \mathrm{~dB}(\mathrm{~ns})$ & \\
\hline
\end{tabular}

BS, binaural summation; ns, nonsignificant.

Table 2 shows the improvements ("Change"; column 7) between studies are rather variable. As a reference, on average a normal-hearing subject shows an MAE between $5^{\circ}$ and $10^{\circ}$. When this subject is acutely plugged unilaterally, a difference in MAE to localizing with both ears open ranges from $60^{\circ}$ to $80^{\circ}$ [Agterberg et al., 2012].

Remarkably, Vyskocil et al. [2017] reported that in one of their 5 subjects, the localization error (expressed as a root-mean-square value) improved from $88^{\circ}$ in unilateral listening condition to $33^{\circ}$ in the aided listening condition (using the Bonebridge). The unilateral listening scores of the other 4 subjects in this particular study were already low, namely $28^{\circ}$ on average (range: $19^{\circ}-37^{\circ}$ ). With the Bonebridge, these 4 children improved their localization score by $8^{\circ}$, on average. Kunst et al. [2008] also presented individual data and, again, a bimodal distribution is seen. Two out of 10 adults and 4 out of 10 children had a poor unilateral localization score with a mean MAE of $95^{\circ}$ and $86^{\circ}$, which decreased with the BCD by $41^{\circ}$ and $19^{\circ}$, respectively. The other 14 subjects had a more favorable pretreatment mean MAE score of $26^{\circ}$ (adults) and $32^{\circ}$ (children). Remarkably, Kunst et al. [2008] showed in 8 out of the 20 subjects, a significant deterioration in the aided compared to the unaided condition $\left(5^{\circ}-24^{\circ}\right)$. Wilmington et al. [1994] found low pretreatment MAE scores of $20^{\circ}$ or better in all of their subjects, with a mean posttreatment improvement of $6^{\circ}$. These data suggest the existence of subgroups, namely subjects with good pretreatment localization scores and with poor pre-treatment scores. A limited improvement after treatment seems to be related to good pretreatment scores. Listeners with poor pretreatment scores (high MAE) demonstrate an obvious improvement in localization after treatment in most cases. Moon et al. [2014] reported a clear improvement in mean correct response rate after canaloplasty in 28 subjects. Preoperative correct response rate (26\%) improved to $59 \%$ and was maintained until 12 months after surgery.

Relatively good localization in subjects with chronic unilateral hearing loss has been reported before and was ascribed to effective use of monaural cues (sound level cues and spectral cues [van Wanrooij and van Opstal, 2004]), which for normal-hearing listeners are only minor cues for azimuth localization.

The Cohen's $d$ effect size, presented in the last column of Table 2 , are for all indicated studies $>0.8$, representing a small effect. Note that although at group level the effect might be small, individual subjects might still demonstrate an obvious improvement when listening in the treated condition.

\section{Binaural Summation}

In 3 papers, binaural summation scores were presented (Table 3). In 2 studies, binaural summation in quiet was tested [Kunst et al., 2008; Kesser et al., 2016], which did not statistically differ from 0 in either study. In these studies, respectively, 30 and $20 \%$ of the subjects showed a negative binaural summation score. This suggests that these subjects were not able to fuse the 2 auditory inputs to a coherent percept. Binaural summation-in-noise was studied by Byun et al. [2015] and Kesser et al. [2016]. They also found that the mean summation scores were not significantly different from 0 . Individual data presented by Kesser et al. [2016] showed that $40 \%$ of their subjects had a negative binaural summation in noise score. Byun et al. [2015] did not present individual data.

Speech Recognition in Noise with Spatially Separated Speech and Noise Sources

Four studies [Wilmington et al., 1994; Kunst et al., 2008; Gray et al., 2009; Byun et al., 2015] reported outcomes of speech tests with spatially separated speech and noise sources. These studies presented data obtained with speech in the front $\left(S_{0}\right)$ and noise presented at the normalhearing side $\left(N_{\mathrm{nh}}\right)$ and, separately, at the impaired side $\left(N_{\text {atresia }}\right)$. Table 4 displays the calculated outcome, $S_{0} N_{n h}-$ $S_{\mathrm{O}} N_{\text {atresia }}$, for both listening conditions (unilateral/bilateral). In the unilateral listening condition (untreated), this difference represents the maximum head shadow effect. After treatment, in the bilateral listening condition, assuming that the ears are equivalent, the score should re- 
Table 4. Head shadow as reported in 4 studies

\begin{tabular}{lllll}
\hline First author & Treatment & $\begin{array}{l}S_{\mathrm{o}} N_{\mathrm{nh}}-S_{\mathrm{o}} N_{\text {atresia }} \\
\text { unilateral listening, dB }\end{array}$ & $\begin{array}{l}S_{\mathrm{o}} N_{\mathrm{nh}}-S_{\mathrm{o}} N_{\text {atresia }} \\
\text { bilateral listening, dB }\end{array}$ & $\begin{array}{l}z \\
\text { scores }\end{array}$ \\
\hline Wilmington et al., 1994 & Atresia repair & 6.8 & 3.7 & 1.3 \\
Kunst et al., 2008, adults & Baha ${ }^{\circledR}$ & 3.8 & 3.0 & 2.4 \\
Gray et al., 2009 & Atresia repair & - & $2.9^{*}$ & - \\
Byun et al., 2015 & Atresia repair & 6.0 & 5.8 & \\
\hline
\end{tabular}

* Using the average data of their Figures $1(3.2 \mathrm{~dB})$ and $2(0.3 \mathrm{~dB})$, the calculated difference is 2.9 .

duce to 0 . However, reported values are clearly above 0 . This indicates that after treatment, acoustic head shadow was not dealt with completely. The calculated Cohen's $d$ effect size of 2 studies showed values $>0.8$, representing a small effect. Looking at individual data, with noise presented toward the side of the normal-hearing ear, $20 \%$ [Gray et al., 2009] and 30\% [Kunst et al., 2008] of the subjects did not show a positive effect of the treatment and some even deteriorated. In the study of Wilmington et al. [1994], subjects showed either an improvement after the surgery or the same results as before the treatment. Byun et al. [2015] did not present individual data.

\section{Discussion}

This review provides an overview of studies addressing posttreatment outcomes in subjects with congenital UCHL. A problem of rehabilitating such subjects is that the input from the treated ear has to integrate (fuse) with the input from the other, normal-hearing ear.

Overall, binaural hearing abilities after treatment, as listed in Tables 2-4, deviate when compared to the performance of normal-hearing subjects. Although at the individual level some patients demonstrate a clear improvement after treatment, at group level the benefit of treatment is limited. There are several possible reasons that could be underlying these poor results. One reason might be the (remaining) asymmetry in hearing thresholds between the ears after treatment. As Table 1 shows, the asymmetry varied from $13 \mathrm{~dB}$ HL up to more than $30 \mathrm{~dB}$ HL. The posttreatment thresholds of 35-40 dB HL (Table 1) are troublesome, which is on average the case for 6 out of the 9 studies. Expressed in "expected word recognition scores," using the "Count-the-dots method" [Killion and Mueller, 2010], posttreatment thresholds of $35 \mathrm{~dB}$ HL result in approximately $70 \%$ word recognition, while thresholds at $20 \mathrm{~dB} \mathrm{HL}$ and lower enable $100 \%$ word recognition. Integration of information from the treated ear with input from the normal ear is probably not achieved in most of the patients in the reviewed studies and presumably explains the poor speech scores obtained in experiments with spatially separated speech and noise sources (Table 4).

Several studies presented individual data and up to $40 \%$ of participating subjects in these studies did not only show no benefit, but even a deterioration was found when comparing post- with pretreatment scores. The posttreatment deterioration in performance deserves more attention. In these specific patients binaural interference, as described by Jerger et al. [2017], might have played a role, which was ascribed to impaired neural processing comparable to amblyopia. Before treatment, subjects with congenital UCHL did not experience binaural hearing and, therefore, might not have a normally developed neural auditory system (as demonstrated in animal models [Moore et al., 1989]). However, in humans with congenital UCHL, in contrast to listeners with unilateral sensorineural hearing loss [Gordon and Kral, 2019], the impaired ear (cochlea) has been stimulated by the subject's own voice via bone and tissue conduction and by loud external sounds, which exceed the hearing threshold of the impaired ear. The question remains if this auditory input on the impaired side, which is less than the normal hearing side, is enough to keep the neural auditory pathway plastic for binaural input at a later stage. In a previous study by Snik et al. [1994], long-term auditory performance (follow-up $>12$ months) in selected subjects with congenital UCHL after successful atresia repair (asymmetry in hearing thresholds after treatment <20 dB; mean: $11 \mathrm{~dB}$ ) has been investigated. Although speech recognition in quiet was the same for the 2 ears, a statistically significant difference was found for the critical SNR of $1.7 \mathrm{~dB}$. Thus, auditory deprivation cannot be ruled out and might have affected the speech-in-noise data, as presented in Tables 3 and 4 .

As a consequence, age at treatment might play a role. Within the age range studied, no effect of age at treatment 
was found [Wilmington et al., 1994]. Snik et al. [1994] came to the same conclusion. Based on their speech-in-noise data, Gray et al. [2009] suggested that the best age for atresia surgery is during adolescence. With regard to auditory maturation, it should be noted that children with age below 6 years have not been included in any of these studies. Therefore, it remains unknown whether or not early treatment ( $<6$ years) might lead to better results. However, more fundamental studies demonstrate that inducing UCHL at birth led to reduction in size of auditory neurons, while inducing UCHL during adulthood did not affect neuronal size [Webster 1983a, b]. Other studies at the morphological and functional level further strengthen the idea of a certain vulnerable period during early auditory maturation [Polley et al., 2013; Liberman et al., 2015; Wang et al., 2019]. Finally, more general studies regarding sensitive and critical periods indicate that experience of asymmetric hearing can result in persistent changes in the auditory system affecting maturation [e.g., Knudsen and Knudsen, 1990].

Nelissen et al. [2015] published data on long-term use of a percutaneous BCD in 53 subjects with congenital UCHL; age at treatment varied from 4 to 54 years. Close to half of them were nonusers (43\%) and one-quarter (23\%) used their BCD sporadically. A significant relationship was found between long-term use and outcomes of binaural tests performed at least 12 weeks after device fitting. Evidently, the nonusers had more problems to fuse the 2 inputs when tested. And it is of course still debatable whether device use affects binaural hearing abilities or vice versa and what the role is of early treatment and coping behavior (e.g., the effective use of monaural spectral cues for horizontal sound localization).

Limitations of this review include the low number of identified relevant studies, the heterogeneity of study protocols, the differences in the method to measure, among others sound localization and the 5 different types of treatments. Therefore, a meta-analysis could not be performed, and it is not possible to indicate the best treatment option. The presented effect scores were poor, ranging from 1.1 to 2.6, indicating large clinical differences at group level between the patients and normal-hearing listeners. These results are in agreement with the conclusion drawn by Danhauer et al. [2010], who reviewed the literature regarding the application of a percutaneous BCD in subjects with congenital UCHL and reported that most patients perceived only small benefit. However, it is also important to note that some patients do demonstrate a clear benefit and the reason for the variation in benefit of treatment remains unexplained.

When comparing outcomes of the BCD to surgical repair, Nadaraja et al. [2013] concluded that implantation of a percutaneous $\mathrm{BCD}$ is the more effective treatment option. However, the best amplification option for congenital UCHL is still indistinctive and treatment is suboptimal. Although in most cases subjects perceive some benefit, this review suggests that benefit might be based on bilateral hearing ( 2 "separate" inputs) rather than on "binaural hearing" (a fused concept). In a minority of subjects, even binaural interference seems to occur. This review does identify possible confounders, namely limitations in auditory neural maturation and a remaining asymmetry in hearing between ears after treatment. Additional research is needed to study the role of these confounders in more depth and to optimize counseling of patients and selection of the best treatment options.

\section{Statement of Ethics}

The research was conducted ethically in accordance with the World Medical Association Declaration of Helsinki, and the authors have no ethical conflicts to disclose.

\section{Conflict of Interest Statement}

The authors have no conflicts of interest to declare.

\section{Funding Sources}

K.V. was funded by FP7-PEOPLE-2013-ITN Marie Curie Initial Training Network iCare. M.J.H.A. was funded by the William Demants og Hustru Ida Emilies Fond and Ad Snik by The European Union Horizon-2020 ERC Advanced Grant 2016 - ORIENT (693400).

\section{Author Contributions}

F.M.S., K.V., and J.D. carried out the search and selection of the papers. Writing was done by F.M.S., K.V., J.D., A.M.J., and M.J.H.A.

\begin{tabular}{|c|c|}
\hline References & $\begin{array}{l}\text { Agterberg MJ, Hol MK, Cremers CW, Mylanus } \\
\text { EA, van Opstal J, Snik AF. Conductive hear- } \\
\text { ing loss and bone conduction devices: re- } \\
\text { stored binaural hearing? Adv Otorhinolaryn- } \\
\text { gol. 2011;71:84-91. } \\
\text { Agterberg MJ, Snik AF, Hol MK, van Wanrooij } \\
\text { MM, van Opstal J. Contribution of monaural } \\
\text { and binaural cues to sound localization in lis- } \\
\text { teners with acquired unilateral conductive } \\
\text { hearing loss: improved directional hearing } \\
\text { with a bone-conduction device. Hearing Res. } \\
\text { 2012;286:9-18. }\end{array}$ \\
\hline
\end{tabular}


Alasti F, Van Camp G. Genetics of microtia and associated syndromes. J Med Genet. 2009; 46(6):361-9.

Avan P, Giraudet F, Buki B. Importance of binaural hearing. Audiol Neurotol. 2015;20(Suppl 1):3-6.

Bess FH, Tharpe AM. Unilateral hearing impairment in children. Pediatrics. 1984;74:206-16.

Blauert J. Spatial hearing. The psychophysics of human sound localization. Cambridge: MIT; 1997.

Bronkhorst AW. The cocktail party phenomenon: a review of research on speech intelligibility in multiple-talker conditions. Acustica. 2000;86:117-28.

Bronkhorst AW, Plomp R. The effect of head-induced interaural time and level differences on speech intelligibility in noise. J Acoust Soc Am. 1988;83(4):1508-16.

Byun H, Moon IJ, Woo SY, Jin SH, Park H, Chung $\mathrm{WH}$, et al. Objective and subjective improvement of hearing in noise after surgical correction of unilateral congenital aural atresia in pediatric patients: a prospective study using the hearing in noise test, the sound-spatialquality questionnaire, and the Glasgow benefit inventory. Ear Hear. 2015;36:183-9.

Carlile S, Martin R, McAnally K. Spectral information in sound localization. Int Rev Neurobiol. 2005;70:399-434.

Cohen J. Statistical power analysis for the behavioral science. Lawrence Erlbaum Associates, Publishers. 1988.

Danhauer JL, Johnson CE, Mixon M. Does the evidence support use of the Baha implant system (Baha) in patients with congenital unilateral aural atresia? J Am Acad Audiol. 2010;21: 274-86.

Davis A, Haggard M, Bell I. Magnitude of diotic summation in speech-in-noise tasks: performance region and appropriate baseline. $\mathrm{Br} J$ Audiol. 1990;24(1):11-6.

Dillon H. Hearing aids. 2nd ed. Thieme; 2012.

Frenzel H, Hanke F, Beltrame M, Steffen A, Schönweiler R, Wollenberg B. Application of the Vibrant Soundbridge ${ }^{\circledR}$ to unilateral osseous atresia cases. Laryngoscope. 2009;119(1): 67-74.

Frenzel H, Sprinzl G, Streitberger C, Stark T, Wollenberg B, Wolf-Magele A, et al. The vibrant soundbridge in children and adolescents: preliminary European multicenter results. Otol Neurotol. 2015;36(7):1216-22.

Gordon K, Kral A. Animal and human studies on developmental monaural hearing loss. Hear Res. 2019;380:60-74

Gray L, Kesser B, Cole E. Understanding speech in noise after correction of congenital unilateral aural atresia: effects of age in the emergence of binaural squelch but not in use of head-shadow. Int J Pediatr Otorhinolaryngol. 2009;73:1281-7.

Jerger J, Silman S, Silverman C, Emmer M. Binaural interference: quo vadis? J Am Acad Audiol. 2017;28(4):266-70.
Kesser BW, Cole ED, Gray LC. Emergence of binaural summation after surgical correction of unilateral congenital aural atresia. Otol Neurotol. 2016;37:499-503.

Kesser BW, Krook K, Gray LC. Impact of unilateral conductive hearing loss due to aural atresia on academic performance in children. Laryngoscope. 2013;123: 2270-5.

Killion MC, Mueller HG. Twenty years later: a new count-the-dots method. Hear J. 2010; 63(1):10-7.

Kitterick PT, Lovett RES, Goman AM, Summerfield AQ. The AB-York crescent of sound: an apparatus for assessing spatial-listening skills in children and adults. Cochlear Implants Int. 2011;12:164-9.

Knudsen EI, Knudsen PF. Sensitive and critical periods for visual calibration of sound localization by barn owls. J Neurosci. 1990;10: 222-32.

Kunst SJ, Leijendeckers JM, Mylanus EA, Hol MK, Snik AF, Cremers CW. Bone-anchored hearing aid system application for unilateral congenital conductive hearing impairment: audiometric results. Otol Neurotol. 2008;29(1):2-7.

Kuppler K, Lewis M, Evans AK. A review of unilateral hearing loss and academic performance: is it time to reassess traditional dogmata? Int J Pediatr Otorhinolaryngol. 2013; 77:617-22.

Leinung M, Zaretsky E, Lange BP, Hoffmann V, Stöver T, Hey C. Vibrant Soundbridge ${ }^{\circledR}$ in preschool children with unilateral aural atresia: acceptance and benefit. Eur Arch Otorhinolaryngol. 2017;274:159-65.

Levitt H, Rabiner LR. Binaural release from masking for speech and gain in intelligibility. $J$ Acoust Soc Am. 1967;42(3):601-8.

Liberman MC, Liberman LD, Maison SF. Chronic conductive hearing loss leads to cochlear degeneration. PLoS One. 2015; 10:e142341.

Lieu JEC. Unilateral hearing loss in children: speech-language and school performance. BENT. 2013;21:107-15.

Lieu JEC, Tye-Murray N, Fu Q. Longitudinal study of children with unilateral hearing loss. Laryngoscope. 2012;122:2088-95.

Magele A, Schoerg P, Stanek B, Gradl B, Sprinzl GM. Active transcutaneous bone conduction hearing implants: systematic review and meta-analysis. PLoS One. 2019;14(9):e0221484.

Moher D, Liberati A, Tetzlaff J, Altman DG;PRISMA Group. Preferred reporting items for systematic reviews and meta-analyses: the PRISMA statement. Ann Intern Med. 2009;151(4): 264-9.

Moon IJ, Byun H, Jin SH, Kwon S, Chung WH, Hong $\mathrm{SH}$, et al. Sound localization performance improves after canaloplasty in unilateral congenital aural atresia patients. Otol Neurotol. 2014;35:639-44.

Moore DR, Hutchings ME, King AJ, Kowalchuk NE. Auditory brain stem of the ferret: some effects of rearing with a unilateral ear plug on the cochlea, cochlear nucleus, and projections to the inferior colliculus. J Neurosci. 1989; 9(4):1213-22.
Nadaraja GS, Gurgel RK, Kim J, Chang KW. Hearing outcomes of atresia surgery versus osseointegrated bone conduction device in patients with congenital aural atresia: a systematic review. Otol Neurotol. 2013;34:13949.

Nelissen RC, Agterberg MJ, Hol MK, Snik AF. Three-year experience with the Sophono in children with congenital conductive unilateral hearing loss: tolerability, audiometry, and sound localization compared to a bone-anchored hearing aid. Eur Arch Otorhinolaryngol. 2016;273:3149-56.

Nelissen RC, Mylanus EA, Cremers CW, Hol MK, Snik AF. Long-term compliance and satisfaction with percutaneous bone conduction devices in patients with congenital unilateral conductive hearing loss. Otol Neurotol. 2015; 36:826-33.

Plomp R, Mimpen AM. Effect of the orientation of the speaker's head and the azimuth of a noise source on the speech reception threshold for sentences. Acustica. 1981;48(5):325-8.

Polley DB, Thompson JH, Guo W. Brief hearing loss disrupts binaural integration during two early critical periods of auditory cortex development. Nat Commun. 2013;4:1-13

Sangen A, Royackers L, Desloovere C, Wouters J, van Wieringen A. Single-sided deafness affects language and auditory development: a case-control study. Clin Otolaryngol. 2017; 42:979-87.

Snik A, Agterberg M, Bosman A. How to quantify binaural hearing in patients with unilateral hearing using hearing implants. Audiol Neurotol. 2015;20(1):44-7.

Snik AFM, Teunissen B, Cremers WR. Speech recognition in patients after successful surgery for unilateral congenital ear anomalies. Laryngoscope. 1994;104(8):1029-34.

Van Wanrooij MM, Van Opstal AJ Contribution of head shadow and pinna cues to chronic monaural sound localization. J Neurosci. 2004;24(17):4163-71.

Vyskocil E, Liepins R, Kaider A, Blineder M, Hamzavi S. Sound localization in patients with congenital unilateral conductive hearing loss with a transcutaneous bone conduction implant. Otol Neurotol. 2017;38:318-24.

Wang X, Liu J, Zhang J. Chronic unilateral hearing loss disrupts neural tuning to soundsource azimuth in the rat primary auditory cortex. Front Neurosci. 2019;13:1-18

Webster DB. Auditory neuronal size after a unilateral conductive hearing loss. Exp Neurol. 1983a;79:130e-140.

Webster DB. Late onset of auditory deprivation does not affect brain stem auditory neuron soma size. Hear Res. 1983b;12:145e-7.

Wilmington D, Gray L, Jahrsdoerfer R. Binaural processing after corrected congenital unilateral conductive hearing loss. Hear Res. 1994; 74:99-114. 